\title{
Modalidades de ensinar e aprender: educação online no curso de graduação em Odontologia
}

Gabrielle Gonsalli Domingues*, Graciela Soares Fonsêca**, Celso Zilbovicius***, Antonio Carlos Frias***, Simone Rennó Junqueira****

\author{
Cirurgiã-Dentista da Prefeitura Municipal de São \\ Paulo \\ ** Professora Adjunta, Curso de Medicina da \\ Universidade Federal da Fronteira Sul (UFFS), \\ campus Chapecó \\ *** Professor Dr. da Faculdade de Odontologia da \\ Universidade de São Paulo (FOUSP) \\ Professora Associada da Faculdade de Odontologia \\ da Universidade de São Paulo (FOUSP)
}

\section{RESUMO}

O trabalho objetivou descrever e qualificar o acesso a computadores e à rede de internet, bem como ao ambiente virtual de aprendizagem (AVA) e suas respectivas ferramentas, por graduandos da Faculdade de Odontologia da Universidade de São Paulo. Estudo transversal, baseado em questionários aplicados em um universo de 715 estudantes, os quais estivessem presentes nas salas de aula das onze turmas dos diferentes anos e turnos. Foram feitas análises de distribuição de frequência e teste de associação no programa EPI-Info v.6.0, medidas pela Razão de Chances (OR - odds ratio). Todos os respondentes $(\mathrm{n}=400)$ possuem computador em casa, 98\% com acesso à internet e 84,5\% utilizam os computadores disponibilizados pela Instituição. Majoritariamente $(89,7 \%)$, os alunos dedicaram mais de duas horas semanais para atividades virtuais. Observouse que os alunos do noturno apresentaram duas vezes mais chances de utilização de computador que alunos do integral. Ao menos uma disciplina que utiliza o AVA (Plataforma Moodle) foi identificada por $96,5 \%$ dos estudantes. Ao acessar o AVA, os alunos declararam ter maior facilidade para acessar a página inicial e os textos disponibilizados. Fóruns e chats foram apontados como ações de menor facilidade de execução. Não houve associação entre o tempo de uso do computador e a avaliação da utilização das ferramentas. Infraestrutura computacional que acompanhe os avanços tecnológicos e a utilização de um AVA com ferramentas acessíveis podem potencializar a incorporação da educação online.

Descritores: Educação à Distância. Educação Superior. Odontologia. Saúde.

\section{INTRODUÇÃO}

A globalização vem transformando e marcando o mundo pelo avanço tecnológico e pela grande disseminação das Tecnologias da Informação e da Comunicação (TIC). Para a sociedade atual, o significado de tempo foi modificado e a distância já não representa uma limitação significativa para a comunicação entre as pessoas ${ }^{1}$.

A educação e o sistema educacional, nesse contexto, foram obrigados a buscar novos direcionamentos, condizentes com o 
seu tempo, auxiliando o estudante a adquirir as condições necessárias para desenvolver-se plenamente como pessoa e cidadão ${ }^{1}$. Para a sociedade do conhecimento, o mais importante não é saber mais e sim melhor. Nesse sentido, faz-se necessário o domínio das estratégias e habilidades de acesso e transformação de conteúdos do saber ${ }^{2}$.

$\mathrm{Na}$ esfera da formação superior em saúde no Brasil, as Diretrizes Curriculares Nacionais $(\mathrm{DCN})^{3}$ - que balizam o ensino de todos os cursos superiores na área da saúde preveem que o aluno deva ser estimulado a "aprender a aprender, a aprender a viver junto, a aprender a ser" pressupostos que se configuram nos pilares da educação ${ }^{4}$.

Contudo, o modelo tradicional de ensino em saúde, pautado na transmissão de informações, consequência dos Relatórios Flexner de 1910 e Gies de 1926, não é capaz de permitir o alcance desse fim. Por isso, tem sido estimulada, entre outras razões, a reorientação dos currículos dos cursos da área da saúde ${ }^{5}$, tentando adequar-se à realidade do país e à política de saúde bucal, no intuito de garantir a capacitação de profissionais com autonomia e discernimento voltado para a integralidade da atenção prestada aos indivíduos, famílias e comunidades ${ }^{6}$.

Para tanto, novas estratégias pedagógicas também devem ser experimentadas. A Educação à Distância (EAD) vem ganhando espaço e impulso. É crescente, no Brasil e em outras partes do mundo, a introdução das ferramentas de informática e de comunicação no sistema educacional $^{7}$. Assim, a EAD aparece como o dispositivo capaz de facilitar o acesso do aluno à informação, tornando-o proativo na construção do conhecimento, sendo agente do seu processo formador e criando seu próprio perfil de aprendizado ${ }^{1}$.
Como colocaram Holanda e colaboradores $^{8}$, a EAD não pode ser mais caracterizada pela distância porque a virtualidade dessa estratégia permite encontros cada vez mais efetivos que potencializam o processo de ensinoaprendizagem. Esses autores acreditam que o termo mais adequado para se referir a esse tipo de estratégia seja 'educação online'.

Nos ambientes online de aprendizagem, o que poderia ser distante se aproxima rompendo a ideia de espaço e tempo, ou seja, essas dimensões - tempo e espaço - são instituídas de acordo com as necessidades e os interesses dos estudantes, ampliando, assim, as possibilidades da educação ${ }^{9}$.

A grande vantagem da educação online encontra-se na possibilidade inerente de respeitar o ritmo de cada aluno, visto que os horários de estudos são designados por ele ${ }^{10-12}$.

Essas características fazem da educação online um modelo coerente com as atuais características da sociedade. O ritmo de vida acelerado e a grande incorporação de tecnologias nos equipamentos de informática e comunicação aliados a exigência cada vez maior de trabalhadores qualificados pelo mercado de trabalho têm levado muitas pessoas a optarem por cursos à distância como forma de obterem aperfeiçoamento profissional. Além disso, essa modalidade permite que pessoas excluídas do modelo tradicional sejam incluídas e tenham seus direitos de acesso à educação e à informação garantidos ${ }^{1}$.

No ambiente de ensino online, a aprendizagem aparece como um processo dinâmico e contínuo de construção ativa do conhecimento e aquisição de habilidades. Essa aprendizagem é assíncrona e é capaz de 
promover a mudança de comportamento, gerar reflexões e críticas sobre o conteúdo estudado, além de estimular a capacidade investigativa dos educandos ${ }^{8}$ e a construção do conhecimento em interação com outros aprendizes ${ }^{13,14}$.

$\mathrm{Na}$ área médico-odontológica, a educação online é utilizada, principalmente, por cursos de pós-graduação e de educação permanente. Para a graduação, a quantidade de trabalhos presentes na literatura referentes à aplicação dessa estratégia pedagógica vem sendo considerada pequena ${ }^{15}$, mas crescente com o passar dos anos.

A educação online pode ser empregada como apoio às atividades presenciais de um curso de graduação tornando-os mais vivos e interessantes, mais vinculados com a nova realidade, de pesquisa e de contatos com os conhecimentos produzidos ${ }^{16}$. A educação online como apoio ao ensino presencial transforma a relação professor-aluno e gera um envolvimento mais efetivo dos estudantes no processo de ensinoaprendizagem ${ }^{17}$.

A Faculdade de Odontologia da Universidade de São Paulo (FOUSP), instituição pública na capital paulista, vem desenvolvendo iniciativas vinculadas à educação online desde a década de 90 . No início, essas ações caracterizaram-se basicamente pela produção de vídeos sobre procedimentos clínicos realizados em pacientes e pela criação de sites que disponibilizavam material educacional como apoio ao ensino presencial. Ambientes Virtuais de Aprendizagem (AVA) passaram a ser usados em 2004 por disciplinas de pósgraduação. Esses ambientes eram, essencialmente, o TelEduc (UNICAMP), o CoL (USP) e o Modular Object-Oriented Dynamic Learning Environment (Moodle) ${ }^{17}$, este último, uma das plataformas de construção colaborativa mundial mais citadas e utilizadas como AVA para facilitar a interação entre alunos e professores ${ }^{1}$.

Com a ideia de criar o Núcleo de Teleodontologia, surgida em 2006, foi possível instalar a Plataforma Moodle na própria unidade favorecendo a disseminação do seu uso entre os docentes, com o apoio da Teleodontologia ${ }^{17}$.

Desde então, a faculdade dispõe de um suporte digital utilizado, crescentemente, por disciplinas da graduação e da pós-graduação, de maneira complementar às atividades presenciais.

Para que a educação online seja efetiva, é necessário avaliar as propostas pedagógicas, atentar-se para o potencial e as limitações desta tecnologia, manter uma integração entre alunos e tutores com o AVA e analisar os resultados obtidos ${ }^{18}$. É imprescindível acompanhar de perto a utilização das TIC no processo educador no sentido de garantir que elas possibilitem o crescimento e o desenvolvimento do potencial humano e científico dos alunos, no entanto, elas não podem ser vistas ou tomadas como um fim em si mesmas ${ }^{1}$.

Ademais, há que se levantar qual o real acesso e domínio que os jovens têm sobre essas novas ferramentas antes de propô-las como estratégia pedagógica.

Embasado por esses argumentos, o trabalho buscou descrever e qualificar o acesso a computadores e à rede de internet, bem como ao ambiente virtual de aprendizagem e suas respectivas ferramentas, por graduandos de odontologia de uma instituição pública no estado de São Paulo, no sentido de potencializar o uso da educação online nos processos de formação da instituição. 


\section{MATERIAL E MÉTODO}

Trata-se de estudo do tipo censo com delineamento transversal, que se utilizou de questionários para coletar os dados. $\mathrm{O}$ projeto foi aprovado pelo Comitê de Ética em Pesquisa da FOUSP seguindo as normas do Conselho Nacional de Saúde 466/12 para pesquisa com seres humanos com o número de aprovação 36/2011.

Foi realizado um piloto com os estudantes do Centro Acadêmico para verificar o tempo dispendido para preenchimento do questionário, assim como para alterar eventuais dúvidas na versão final.

Os sujeitos do estudo foram alunos de graduação, matriculados nos cursos integral e noturno. O critério para inclusão na amostra se deu em decorrência do aceite de participação, por meio da assinatura do Termo de Consentimento Livre e Esclarecido.

Dois dos pesquisadores estiveram presentes em sala de aula, em todas as turmas (cinco turmas do curso integral e seis turmas do curso noturno), num possível universo de 715 estudantes. Todos os presentes em sala de aula foram convidados a participar (excluídos aqueles que responderam ao piloto) e esclarecidos quanto aos procedimentos e objetivo da pesquisa.

O questionário foi organizado por questões de múltipla escolha versando sobre o uso de computador e sobre as atividades de educação online desenvolvidas na instituição. As variáveis foram distribuídas em dois grupos: uso do computador e qualificação do uso de ferramentas da plataforma Moodle.

Os dados foram digitados em planilha do Microsoft Office Excel 2007®, analisados mediante distribuição de frequência e discutidos de acordo com a literatura científica. No Programa EPI-Info, versão 6.0, foram feitos testes de associação, medidos pela Razão de Chances (OR-odds ratio) e definindo-se $o$ ponto de corte para significância estatística em 5\% ( $\mathrm{p}<0,05)$.

Buscou-se associação entre a quantidade de horas de uso do computador e o tipo de curso (se integral ou noturno), bem como com a avaliação do uso de ferramentas da Plataforma Moodle (dicotomizadas nas categorias ruim/regular para os escores de $1 \mathrm{a}$ 3 e bom/ótima para os escores 4 e 5).

\section{RESULTADOS}

Do total de alunos dos cursos de odontologia (integral e noturno) matriculados no momento da intervenção, 400 responderam ao questionário, o que correspondeu a 55,9\% do universo. Observou-se que um número maior de alunos do curso integral $(54,5 \%)$, que possui maior número de vagas, respondeu ao inquérito. Essa distribuição, também em função do ano de ingresso pode ser observada na tabela 1 .

Todos os alunos que responderam ao questionário possuem computador em suas residências, 98,0\% dos quais com acesso à internet de alta velocidade. O hábito de usar lan house foi apontado por apenas $1 \%$ dos entrevistados. A tabela 2 ilustra a quantidade de alunos que se utiliza dos espaços criados pela instituição para o uso de computadores, como a sala Pró-aluno e a Biblioteca. Mesmo com suporte eletrônico em casa, é grande a quantidade de alunos que necessita usar desses recursos no ambiente escolar $(84,5 \%)$.

A tabela 3 traz a distribuição dos alunos em função do tempo médio gasto por dia no uso do computador. Grande parte dos alunos $(44,2 \%)$ dedica até duas horas semanais ao uso da máquina. Uma porcentagem menor $(32,2 \%)$ direciona de 3 a 4 horas para essa atividade e 13,2\% utilizam o computador por mais de 4 horas. Para $10 \%$ 
dos alunos, a frequência do uso do recurso foi semanal, sendo que os valores variaram de 1 a 3 horas para $45 \%$ deles e de 4 a 7 horas para $50 \%$.

Procurou-se avaliar se havia influência do tipo do curso (integral ou noturno) na frequência do uso de computador, cuja hipótese aventava para o fato dos alunos do noturno terem mais tempo para isso (Tabela $4)$.

Confirmou-se que os alunos do curso noturno têm, pelo menos, duas vezes mais chances de se dedicar ao uso diário de dispositivos eletrônicos.

Tabela 1- Composição da amostra, segundo o ano de ingresso dos alunos e o curso. FOUSP, 2011.

\begin{tabular}{lcccc}
\hline \multirow{2}{*}{ Ano de Ingresso } & \multicolumn{2}{c}{ Integral } & \multicolumn{2}{c}{ Noturno } \\
& $\mathrm{n}$ & $\%$ & $\mathrm{n}$ & $\%$ \\
\hline 2005 & 0 & 0 & 5 & 2,74 \\
2006 & 1 & 0,45 & 38 & 20,8 \\
2007 & 4 & 1,83 & 25 & 13,7 \\
2008 & 73 & 33,4 & 26 & 14,2 \\
2009 & 51 & 23,3 & 28 & 15,3 \\
2010 & 38 & 17,4 & 24 & 13,1 \\
2011 & 51 & 23,3 & 36 & 19,7 \\
\hline Total & 218 & 100 & 182 & 100 \\
\hline
\end{tabular}

Tabela 2 - Frequência no uso de computadores disponibilizados pela Instituição de Ensino. FOUSP, 2011.

\begin{tabular}{lcc}
\hline Uso de Computadores na FOUSP & $\mathbf{n}$ & $\mathbf{\%}$ \\
\hline Não & 61 & 15,2 \\
Sim & 338 & 84,5 \\
Não respondeu & 1 & 0,25 \\
\hline Total & 400 & 100 \\
\hline
\end{tabular}

Tabela 3 - Número de alunos segundo o tempo médio gasto no computador (independente do tipo de atividade e local de uso). FOUSP, 2011.

\begin{tabular}{lcc}
\hline Tempo de uso & $\mathbf{n}$ & $\mathbf{\%}$ \\
\hline Até 2 horas/dia & 177 & 44,2 \\
De 3 a 4 horas/dia & 129 & 32,2 \\
Mais de 4 horas/dia & 53 & 13,2 \\
Uso semanal & 40 & 10,0 \\
Não respondeu & 1 & 0,25 \\
\hline Total & 400 & 100 \\
\hline
\end{tabular}


Tabela 4 - Associação do tempo de uso diário de computadores, em função do curso. FOUSP, 2011.

\begin{tabular}{|c|c|c|c|c|}
\hline \multirow[t]{2}{*}{ Curso } & \multicolumn{2}{|c|}{ Uso diário do computador } & \multirow[t]{2}{*}{ OR } & \multirow[t]{2}{*}{$P$} \\
\hline & Mais de $2 \mathrm{~h}$ & Até $2 \mathrm{~h}$ & & \\
\hline Noturno & 100 & 66 & 2,05 & $0,0011^{*}$ \\
\hline Integral & 82 & 111 & $(1,32-3,20)$ & \\
\hline
\end{tabular}

Apesar da existência da plataforma Moodle e da sua incorporação crescente às disciplinas da graduação, $10 \%$ dos alunos não relataram o uso dessa plataforma durante sua formação. De maneira contrária, a maioria deles $(89,7 \%)$ identificou de 1 a 6 disciplinas que se utilizaram do AVA, em especial a Plataforma Moodle.

Foi solicitado aos alunos que atribuíssem notas para algumas das ferramentas disponibilizadas nesse ambiente, tendo em vista o grau de facilidade para a participação, o que pode melhorar a adesão (tabela 5). A avaliação sobre a facilidade de acesso à página inicial foi bastante heterogênea e distribuída de forma similar entre cada escore, com ligeira superioridade para a máxima facilidade $(25,7 \%)$. A disponibilidade de textos foi avaliada, em sua maioria, como de fácil utilização sendo que a maioria dos alunos atribuíram os dois maiores graus de facilidade $(56,8 \%)$ para acessar os textos. Já a participação em chats e fóruns foi, predominantemente, avaliada nos scores mais baixos, entre 1 e 3 , somando $74,9 \%$ para chats e $66,5 \%$ para fóruns.

A maioria das disciplinas $(66 \%)$ se utiliza de outros recursos para aprendizagem via internet, sendo a disponibilidade de matéria via correio eletrônico $(57,5 \%)$ e a busca de materiais de estudos por meio eletrônico $(37,8 \%)$ as mais citadas; o uso de redes sociais $(9,5 \%)$ e de teleconferências $(7,3 \%)$ também foram lembradas.

Não houve associação entre o tempo de uso do computador e a avaliação do acesso às ferramentas da Plataforma Moodle (tabela $6)$.

Tabela 5 - Atribuição de nota de 1 a 5 (de nenhuma facilidade à máxima facilidade) para o uso de ferramentas da Plataforma Moodle. FOUSP, 2011.

\begin{tabular}{lccccccccccc}
\hline Atividades & $\mathbf{1}$ & & $\mathbf{2}$ & & $\mathbf{3}$ & $\mathbf{4}$ & & $\mathbf{5}$ & \multicolumn{2}{c}{ Total } \\
& $\mathrm{n}$ & $\%$ & $\mathrm{n}$ & $\%$ & $\mathrm{n}$ & $\%$ & $\mathrm{n}$ & $\%$ & $\mathrm{n}$ & $\%$ & $\mathrm{n}$ \\
\hline $\begin{array}{l}\text { Acesso à página } \\
\text { Principal }\end{array}$ & 76 & 19,5 & 67 & 17,2 & 80 & 20,5 & 66 & 16,9 & 100 & 25,7 & 389 \\
$\begin{array}{l}\text { Disponibilização } \\
\text { de Textos }\end{array}$ & 24 & 6,23 & 52 & 13,5 & 90 & 23,3 & 120 & 31,1 & 99 & 25,7 & 385 \\
Chats & & & & & & & & & & & \\
Fóruns & 72 & 23,3 & 76 & 24,5 & 84 & 27,1 & 52 & 16,8 & 25 & 8,09 & 309 \\
& 65 & 19,8 & 62 & 18,9 & 91 & 27,8 & 73 & 22,3 & 36 & 11,0 & 327 \\
\hline
\end{tabular}


Tabela 6 - Associação da avaliação do uso de ferramentas da Plataforma Moodle em função das horas de acesso ao computador. FOUSP, 2011.

\begin{tabular}{llcccc}
\hline Variável & Avaliação & $\begin{array}{c}\text { Até 2 } \\
\text { h/dia }\end{array}$ & $\begin{array}{c}\mathbf{+ 2} \\
\text { h/dia }\end{array}$ & OR & $\boldsymbol{p}$ \\
\hline $\begin{array}{l}\text { Acesso à Página } \\
\text { Principal }\end{array}$ & Ruim/Regular & 97 & 101 & 0,96 & 0,9375 \\
& Bom/Ótima & 75 & 75 & $(0,61-1,50)$ & \\
$\begin{array}{l}\text { Disponibilização de } \\
\text { Textos }\end{array}$ & Ruim/Regular & 71 & 77 & 0,92 & 0,7914 \\
& Bom/Ótima & 99 & 99 & $(0,59-1,44)$ & \\
Chats & Ruim/Regular & 106 & 104 & 0,71 & 0,2795 \\
& Bom/Ótima & 39 & 27 & $(0,39-1,28)$ & \\
\hline & Ruim/Regular & 99 & 99 & 0,94 & 0,8979 \\
Fóruns & Bom/Ótima & 49 & 46 & $(0,56-1,58)$ & \\
\hline
\end{tabular}

\section{DISCUSSÃO}

A maioria dos alunos (98\%) que participaram do estudo possuem computador disponível no domicílio com acesso à internet de alta velocidade. Resultados semelhantes foram encontrados por Arieira e colaboradores $^{1}$ em que mais de $80 \%$ dos acadêmicos que participaram da investigação afirmaram possuir computador em casa. Esses dados reforçam a viabilidade de inclusão de estratégias de educação online no sentido de agregar no processo de formação, já que o recurso necessário para sua incorporação é acessível para o corpo discente.

No entanto, é importante frisar que podem existir dificuldades ou limitações na utilização da educação online, como a falta de acesso à tecnologia e a falta de preparo para lidar com ela ${ }^{19}$.

É fato que, na ocasião de aplicação do questionário, ainda não era disponibilizado o acesso à rede sem fio nas dependências da instituição, e que aparelhos móveis também não eram usuais entre os alunos.

Um grande número de alunos afirmou necessitar dos computadores disponibilizados pela Faculdade para desenvolver suas atividades, o que faz da mesma o principal lócus pedagógico para a modalidade de ensino virtual.

Isso reforça a imprescindibilidade de garantir infraestrutura computacional e de acesso sem fio à rede para uso da comunidade acadêmica na unidade de ensino. Sem dúvida, a disponibilidade do recurso em ambiente escolar potencializa a educação online uma vez que aumenta as chances de acesso aos AVA.

Uma variável que os autores julgaram fundamental para se pensar em propor atividades que preveem o uso da informática e da internet, diz respeito ao tempo que os 
jovens dispensam com esses recursos. Vale ressaltar que o consumo das tecnologias da nova mídia, principalmente a internet, é expressivo entre os jovens. A atual geração de adolescentes não "adotou" esses recursos porque eles sempre fizeram parte de suas vidas, ou seja, ela já nasceu em um mundo permeado pelo uso da internet ${ }^{21}$.

Nesse estudo, ficou claro que os alunos dedicam uma parte considerável do seu tempo ao uso do computador, sendo que $76,4 \%$ utilizam a máquina, pelo menos, duas horas por semana, o que indica a afinidade dos sujeitos do estudo para utilização de computadores e, possivelmente, para acesso à internet. Corroborando com esses dados, um estudo desenvolvido na Universidade de Brasília, evidenciou que $41,9 \%$ dos graduandos em Enfermagem dedicam de 2 a 3 horas por semana para acesso à internet ${ }^{22}$.

Contudo, o estudo evidenciou que alunos matriculados no curso noturno apresentam mais chances de se dedicar ao uso do computador. Esse achado revela que a abordagem e a organização da educação online devem diferenciar-se em decorrência do tipo de curso dos alunos (integral ou noturno).

De maneira semelhante ao que apontou o trabalho de Arieira e colaboradores $^{1}$, o perfil dos estudantes da presente investigação é propício para exploração do potencial da Educação online, uma vez que eles podem ser considerados proativos, conhecem as ferramentas digitais e possuem disponibilidade de computadores conectados à internet.

Diversos cursos de graduação em saúde vêm incorporando ferramentas digitais como técnica complementar ao processo de ensino-aprendizagem ${ }^{8,22,23}$. No entanto, chama atenção a pequena quantidade de discipli- nas da Instituição que utilizam a Plataforma Moodle. A maior parte dos estudantes $(89,7 \%)$ afirmou que apenas de 1 a 6 disciplinas incluem a plataforma na sua metodologia de ensino.

No segundo semestre de 2014, estavam inscritas na Plataforma Moodle Teleodontologia cerca de 15 disciplinas da graduação, de um total de 63, algumas inscritas duas vezes por serem disponibilizadas para os cursos integral e noturno.

Esse dado leva à reflexão sobre a necessidade de sensibilização do corpo docente com relação ao uso das ferramentas online para potencializar o processo de ensino-aprendizagem. Reconhece-se que a mediação pedagógica no AVA exige preparo e qualificação ${ }^{24}$. O mediador (docente e/ou tutor) é fundamental para o sucesso da educação online 1 e não poderá desempenhar bem essa função se não acreditar nos benefícios dessa modalidade educacional. Ao incorporar o uso da educação online, torna-se necessário agregar novas competências e novos mecanismos de comprometimento e envolvimentos dos atores ${ }^{17}$.

É preciso compreender que não se trata de escolher a educação online em detrimento do ensino presencial, mas, sim de utilizar as TIC como apoio e complementação, inseridas em um contexto planejado e estruturado, com uma abordagem metodológica suportada por uma linha pedagógica própria ${ }^{17}$.

O problema não é exclusivo dos cursos de odontologia. Um trabalho que analisou o uso da Plataforma Moodle em disciplinas específicas de um curso de enfermagem, por exemplo, apontou a necessidade de se potencializar o uso desse AVA em um número maior de disciplinas ${ }^{23}$.

O estudo levantou a possibilidade de haver problemas de utilização da própria 
Plataforma Moodle, o que dificultaria a sua incorporação efetiva. Ao avaliar o grau de facilidade de uso da plataforma Moodle em diversas ferramentas, ficou evidente a facilidade que os alunos encontram para acessar textos de apoio às disciplinas.

No entanto, ao avaliar o uso de fóruns e de chats, a maior porcentagem dos alunos apontou até o grau 3 de facilidade de uso (74,9\% para os chats e $66,5 \%$ para os fóruns) o que permite inferir que ou essas estratégias não estão sendo incorporadas nas disciplinas ou os estudantes não participam efetivamente delas. Assim, esse resultado parece indicar que a utilização da Plataforma Moodle ainda se apresenta como busca a um repositório de material didático e há pouca interação pedagógica e construção de conhecimento dentro do ambiente.

$\mathrm{Na}$ avaliação feita por Arieira e colaboradores $^{1}$ com relação ao uso da plataforma Moodle por estudantes, houve grande aprovação, sendo que apenas 3,3\% dos acadêmicos que participaram do estudo consideraram a ferramenta ruim. Outro trabalho, desenvolvido na Escola de Enfermagem da USP mostrou que os alunos que cursaram a disciplina da graduação que se utilizava do Moodle como recurso auxiliar avaliaram a plataforma de uma maneira muito positiva $^{23}$

Segundo os alunos, a grande parte das disciplinas utiliza outros recursos de aprendizagem via internet, como a disponibilização de conteúdo via correio eletrônico e a busca de material de estudo em sítios eletrônicos. Essa constatação reforça a subutilização da Plataforma Moodle na instituição.

A principal limitação desse estudo refere-se à restrição feita, no questionário, ao uso da internet por meio de computadores.
Sabe-se que o desenvolvimento tecnológico das últimas décadas foi responsável por gerar uma gama de novos dispositivos capazes de acessar à internet, como celulares e tablets. No entanto, essa opção foi feita com o objetivo de diminuir os vieses do estudo, uma vez que, para fins didáticos, na maioria das vezes, o computador é o dispositivo mais utilizado pelos jovens. Outra limitação se refere ao fato de não podermos assegurar que o tempo dispendido ao uso do computador garante o acesso ao AVA, e não há garantias de que ocorra uma ação pedagógica se o mesmo for acessado.

\section{CONSIDERAÇÕES FINAIS}

$\mathrm{O}$ acesso à computadores e à rede de internet em casa ou na unidade de ensino se mostrou alto nesse estudo, o que demonstra um efetivo potencial para a utilização da educação online na formação dos estudantes.

Há, na instituição, suporte para professores e alunos utilizarem ambientes virtuais de aprendizagem, entretanto, ficou evidente a subutilização da Plataforma Moodle da modalidade EAD, complementar às disciplinas presenciais.

Para potencializar a incorporação da educação online torna-se necessário melhorar a infraestrutura computacional da unidade, acompanhando os avanços tecnológicos, bem como utilizar plataformas que garantam um acesso rápido e fácil aos diversos recursos das mesmas, propiciando uma diversidade de estratégias de ensino virtual.

\section{ABSTRACT \\ Modes of teaching and learning: online education in undergraduate course in Dentistry}

This study aimed to describe and qualify the access to computers, internet network and the virtual learning environment (VLE) and its 
tools, by undergraduate students of the Faculty of Dentistry, University of São Paulo. Cross-sectional study based on questionnaires applied in a universe of 715 students, who were present in the eleven classrooms of daytime and nightime courses. Frequency distribution analysis were made and association test in the EPI-Info v.6.0 program, measured by odds ratio (OR). All respondents $(n=400)$ have a computer at home, $98 \%$ with internet access. $84.5 \%$ use the computers provided by the institution. The majority $(89.7 \%)$ of students spent more than two hours per week in virtual activities. It was observed that the nightime course students were twice as likely to use a computer than daytime course students. $96.5 \%$ of the students identified at least one discipline that uses the VLE (Moodle platform). When accessing the AVA, the students claimed to have easier access to the home page and available texts. Forums and chat rooms were cited as less easy to use. There was no association between the time of use of computer and the evaluation of tools utilization. Computing infrastructure that accompanies technological advances and the use of a VLE with accessible tools can enhance the incorporation of online education. Descriptors: Distance Education. Higher Education. Dentistry. Health.

\section{REFERÊNCIAS}

1. Arieira JO, Dias-Arieira CR, Fusco JPA, Sacomano JB, Bettega MOP. Avaliação do aprendizado via educação a distância: a visão dos discentes. Ensaio: Aval Pol Públ Educ. 2009 Abr/Jun;17(63):313340. http://www.scielo.br/pdf/ensaio/ v17n63/v17n63a07.pdf

2. Fernández NR. Fundamentos del proceso educativo a distancia: enseñanza, aprendizaje y evaluación. RIED. 2014;17 (2):75-93.

3. Brasil. Ministério da Saúde. Ministério da Educação. Resolução CNE/CNS 3/2002.
Diretrizes Curriculares Nacionais do Curso de Odontologia. Diário Oficial da União, Brasília, 4 de março de 2002. Seção 1, p.10, Brasília, 2002.

4. Delors J. Educação: um tesouro a descobrir. São Paulo: Cortez, UNESCO, MEC, 1999. 288p.

5. Brasil. Ministério da Saúde. Ministério da Educação. Secretaria de Gestão do Trabalho e da Educação na Saúde. Programa Nacional de Reorientação da Formação Profissional em Saúde - Prósaúde. Brasília, 2005, 80p. Disponível em http://www.saude.gov.br/sgtes. Acesso em 12 de abril de 2007.

6. Terada RSS, Nakama L. A implantação das Diretrizes Curriculares Nacionais de Odontologia: a experiência de Maringá. São Paulo: Hucitec, Rede Unida, ABENO, 2004. 180p.

7. Macedo MCS, Antoniazzi JH. The benefits and newly required practices brought about by innovations in the communication between professionals. Braz Oral Res. 2009;23(2):99-100. http://www.ncbi.nlm.nih.gov/pubmed/19 684940

8. Holanda VR, Pinheiro AKB, Pagliuca LMF. Aprendizagem na educação online: análise de conceito. Rev Bras Enferm [online]. 2013;66(3):406-411. http:// www.scielo.br/pdf/reben/v66n3/a16v66n $\underline{\text { 3.pdf }}$

9. Dias DC, Cassiani SHB. Educação de Enfermagem sem distâncias: uma ruptura espaço/temporal. Rev Esc Enferm USP [online]. 2004;38(4):467-474. http:// www.scielo.br/pdf/reben/v66n3/a16v66n 3.pdf

10. Syed MR. Diminishing the distance in distance education. IEEE Multimedia. 2001;8(3):18-21. 
http://ieeexplore.ieee.org/stamp/stamp.js $\mathrm{p}$ ?tp=\&arnumber $=939996$

11. Franco MA, Cordeiro LM, Castillo RAF. $\mathrm{O}$ ambiente virtual de aprendizagem e sua incorporação na Unicamp. Educ Pesqui [online]. 2003;29(2):341-353. http:// www.scielo.br/pdf/ep/v29n2/a11v29n2.p $\underline{\mathrm{df}}$

12. Demo P. Universidade, aprendizagem e avaliação: horizontes reconstrutivos. Porto Alegre: Editora Mediação, 2005. $157 \mathrm{p}$.

13. Martins TYC, Ribeiro RC, Prado C. Transdisciplinaridade na educação à distância: um novo paradigma no ensino de Enfermagem. Rev Bras Enferm [online]. 2011;64(4):779-782.

14. Medeiros Z, Coelho ML. Encontros presenciais e ambiente virtual de aprendizagem na formação em docência do ensino superior. Rev Docência Ens Sup. 2004 Out;4:47-72.

15. Corrêa L. Análise da Mudança de Paradigma do Ensino de Graduação em Patologia na Odontologia: Proposta de Ensino-Aprendizado a Distância Via Internet. 2001. 205f. Tese (Doutorado em Patologia Bucal) - Faculdade de Odontologia, Universidade de São Paulo, São Paulo, 2001. http://www.teses. usp.br/teses/disponiveis/23/23141/tde-23 042007-155640/pt-br.php

16. Masseto MT. Competência Pedagógica do Professor Universitário. São Paulo: Summus Editorial, 2003. 194p.

17. Haddad AE, Skelton-Macedo MC. Teleodontologia na formação dos profissionais de saúde. In: Mathias I, Alexandra M. (Org). Gold book [recurso eletrônico]: Inovação tecnológica em educação e saúde. Rio de Janeiro: Ed. UERJ, 2012. p. 173-206.
18. Laguardia J, Portela MC, Vasconcellos MM. Avaliação em ambientes virtuais de aprendizagem. Educ Pesq. 2007;33(3): 513-30. http://www.scielo.br/pdf/ep/ v33n3/a09v33n3.pdf

19. Oliveira MAN. Educação à Distância como estratégia para a educação permanente em saúde: possibilidades e desafios. Rev Bras Enferm [online]. 2007;60(5):585-589. http://www.scielo. br/pdf/reben/v60n5/v60n5a19.pdf

20. Morita MC, Haddad AE, Araújo ME. Perfil atual e tendências do cirurgiãodentista brasileiro. Dental Press International: Maringá, 2010.

21. Barcelos RH. Nova mídia, socialização e adolescência: um estudo exploratório sobre o consumo de novas tecnologias de comunicação pelos jovens. 2010. $232 \mathrm{f}$. Dissertação (mestrado) - Universidade Federal do Rio Grande do Sul, Porto Alegre, 2010. http://www.lume. ufrgs.br/handle/10183/24512

22. Peixoto HM, Peixoto MM, Alves ED. Estratégias de aprendizagem utilizadas por graduandos e pós-graduandos em disciplinas semipresenciais da área de saúde. Rev Latino-Am Enf [online]. 2002;20(3):551-8.

http://repositorio.unb.br/bitstream/10482 /12434/1/ARTIGO_EstrategiasAprendiz agemUtilizadas.pdf

23. Prado C. Santiago LC, Silva JAM, Pereira IM, Leonello VM, Otrenti E, Peres HHC, Leite MMJ. Ambiente virtual de aprendizagem no ensino de Enfermagem: relato de experiência. Rev Bras Enferm [online]. 2012;65(5):862-6. http://www.scielo.br/pdf/reben/v65n5/22 . $\mathrm{pdf}$

24. Mendes IAA, Laranjo JC, Araújo FS. A formação continuada em tutoria a partir 
da interação no ambiente virtual. Rev Docência Ens Sup. 2014; 4:73-100. https://seer.ufmg.br/index.php/rdes/articl e/view/924/714
Correspondência para:

Simone Rennó Junqueira

e-mail: srj@usp.br

Faculdade de Odontologia da

Universidade de São Paulo (FOUSP)

Av. Prof. Lineu Prestes, 2227 05558-000

Cidade Universitária - São Paulo/SP 